\title{
A comparative study of fluorescent microscopy with Ziehl-Neelsen staining and culture for the diagnosis of pulmonary tuberculosis
}

\section{Laifangbam $\mathrm{S}^{1}$, Singh $\mathrm{HL}^{2}$, Singh NB${ }^{3}$, Devi KM${ }^{1}$, Singh $\mathrm{NT}^{4}$}

${ }^{1}$ Post-graduate student, ${ }^{2}$ Professor and Head, Department of Microbiology, Kathmandu Medical College Teaching Hospital, Sinamangal, Kathmandu, ${ }^{3}$ Professor and Head, Microbiology Department, ${ }^{4}$ Professor and Head, Respiratory Diseases Department, Regional Institute of Medical Sciences, Imphal, Manipur, India

\begin{abstract}
Background: For developing countries with a large number of cases and financial constraints, evaluation of rapid and inexpensive diagnostic methods has great importance. The bacilli in the sputum can be detected microscopically by $\mathrm{ZN}$ stain and fluorochrome stain.

Objectives : To study the efficacy of fluorescence microscopy in the diagnosis of pulmonary tuberculosis in comparison to Ziehl-Neelsen staining and culture of sputum samples from patients suspected of pulmonary tuberculosis.

Materials and methods : 306 sputum samples collected from 102 patients suspected of pulmonary tuberculosis were processed by the Petroff's method, and subjected to Ziehl-Neelsen staining (ZN), fluorescent Auramine-O staining (AO) and culture on modified Lowenstein-Jensen media (gold standard) for detection of Mycobacterium tuberculosis. Positive smears were graded according to Forbes BA et al, and culture isolates were biochemically tested for confirmation of species.

Results : Out of 102 patients, 44.1\%, 71.6\% and 70\% were found positive by ZN, AO and culture respectively. AO was found to be superior to $\mathrm{ZN}$ on several aspects. The difference in their case detection rates was statistically significant $\left(\chi^{2}\right.$ $=24.93, \mathrm{p}<0.001)$. AO was also able to detect more pauci-bacillary cases than ZN. There was more agreement between culture and fluorescence microscopy $(95.1 \%)$ than with ZN microscopy $(69.6 \%)$. The percentage of false negative by AO staining was only $2.78 \%$ which was in sharp contrast to that of $\mathrm{ZN}(40.27 \%)$.

Conclusion: The better case detection rates of AO over ZN were comparable to those found by several studies. Since screening was done under lower power of magnification (400x), fluorescence microscopy has been found to be less time consuming as compared to $\mathrm{ZN}$ method $(1000 \mathrm{x})$ in the diagnosis of tuberculosis. The tubercle bacilli stood out as bright objects against a dark background in fluorescence microscopy which makes them easily identifiable hence causing less eye-strain. The efficacy of fluorescence microscopy proved to be much higher than conventional light microscopy and comparable to that of culture.
\end{abstract}

Key words: Ziehl-Neelsen staining, Mycobacterium tuberculosis, Auramine-O

$\mathrm{T}$ uberculosis is one of the oldest known diseases of mankind. Yet, the disease is still at large. For developing countries with a large number of cases and financial constraints, evaluation of rapid and inexpensive diagnostic methods like demonstration of acid-fast bacilli (AFB) in smears has great importance.

The bacilli in the sputum can be detected microscopically by $\mathrm{ZN}$ stain and fluorochrome stain. In fluorochroming, a direct chemical interaction occurs between the fluorescent dye and a component of the bacterial cell; this interaction is the same as occurs with the stains used in light microscopy ${ }^{1}$. But, a significant difference in the sensitivity of the two methods has been reported ${ }^{2}$.

Confirmed diagnosis cannot be made by ESR, Mycodot test and even by ELISA technique. Newer tests, based on advanced technology, such as, PCR, gas liquid chromatography and luciferase activity, are costly and only available in few laboratories, and further, specificity is not $100 \%$. The results are quite variable from centre to centre. The diagnosis of pulmonary tuberculosis by chest X-ray is unreliable.

In this study, the staining methods are compared and evaluated taking culture on modified LowensteinJensen (LJ) medium, as "gold standard"3.

Correspondence

Dr. H. Lokhendro Singh,

Professor and Head, Department of Microbiology

Kathmandu Medical College,

Sinamangal, Kathmandu

E-mail: lokhendro_h@yahoo.co.in 


\section{Materials and methods}

This comparative study was conducted in the Department of Microbiology, Regional Institute of Medical Sciences, Imphal, Manipur, India, from January 2004 to June 2005 , on the sputum specimens of 102 patients suspected of pulmonary tuberculosis.

Patient inclusion and exclusion criteria: Patients attending the Respiratory Diseases Department OPD and the DOTS Centre, RIMS, and having fever, night sweats, cough for more than 3 weeks with sputum, loss of appetite, loss of weight, chest pain, haemoptysis and/ or radiological evidence of tuberculosis were included. Those cases who have not taken a course of antibiotics, known cases of carcinoma lung and paediatric cases were excluded. Those unable to produce at least $5 \mathrm{ml}$ of mucopurulent sputum were also excluded.

Sample collection: 3 sputum samples were collected on 2 consecutive days from each patient - spot specimen on the first day, one early morning and one spot specimen on the second day. Samples were collected in clean, sterile, leak-proof, wide-mouth containers.

At the time of sample collection, a Proforma was used to collect data about the patients. On obtaining the study results, the data was completed and analysed statistically using $\chi^{2}$ and p-value.

The processings of the samples were carried out in a biosafety cabinet. Each sample was processed by the Petroff's method 4 and subjected to Ziehl-Neelsen (ZN) staining, Fluorescent Auramine-O (AO) staining and culture on modified Lowenstein-Jensen medium.

Smear reporting is done according to Forbes BA et al. ${ }^{1}$

\section{Acid -fast smear reporting}

Smears are examined using a light microscope scanning at least 300 oil immersion fields before reporting a smear as negative. ${ }^{1,5}$

AFB stain bright pink to red, beaded or barred forms are seen in Mycobacterium tuberculosis while the tissues cells and other organisms are stained blue.

Auramine-o smear reporting

The fluorescence microscope used is the Axiostar plus Transmitted-Light Microscope with an attached Epifluorescence illuminator manufactured by Zeiss and is fitted in a dark room.

The film is examined with a $40 x$ objective and a $10 x$ eyepiece. The tubercle bacilli are seen as yellow luminous organisms in a dark field (Figure 1).

When fluorescent bacilli have been detected the smear was re-stained with ZN stain for confirmation under oilimmersion examination. ${ }^{3}$

For the present study, $2+, 3+$, and $4+$ were classified as multibacillary and $1+$ as paucibacillary. Doubtful is considered negative.

\begin{tabular}{|c|c|c|}
\hline $\begin{array}{l}\text { Number of AFB seen } \\
\text { Fuchsin stain (1000x } \\
\text { magnification) }\end{array}$ & $\begin{array}{c}\text { Number of AFB seen } \\
\text { Fluorochrome stain (400x } \\
\text { magnification) }\end{array}$ & Report \\
\hline 0 & 0 & No AFB seen \\
\hline $1-2 / 300$ fields & $1-2 / 70$ fields & Doubtful \\
\hline $1-9 / 100$ fields & 2-18/50 fields & $1+$ \\
\hline $1-9 / 10$ fields & 4-36/10 fields & $2+$ \\
\hline 1-9/ field & 4-36/ field & $3+$ \\
\hline$>9 /$ fields & $>36 /$ field & $4+$ \\
\hline
\end{tabular}

Smear reporting according to Forbes BA et al.

Culture

Modified Lowenstein-Jensen media were prepared inhouse. After processing, $0.25 \mathrm{ml}$ of the sediment from the decontaminated sputum specimen was inoculated on to the surface of two modified LJ media ${ }^{6}$. After spreading the inoculum over the surface of the slant, the tubes were incubated at $37^{\circ} \mathrm{C}$ and left in the slanted position for 7 days to permit even distribution of the inoculum over the entire surface of the medium. The tubes are then placed upright and incubation continued for 8 weeks. The caps of the bottles were loosened once weekly at the time of inspection for growth. A negative report was given if no growth appeared after 8 weeks ${ }^{7}$.

Observation of colony morphology:

The colonies of $M$. tuberculosis were rough, raised, thick, with a nodular or wrinkled surface and an irregular thin margin (Fig 2). They were non-pigmented (off-white to faint buff), tenacious and not easily emulsified. 
Identification of Mycobacterium tuberculosis:

- Rate of growth.

- Culture growth characteristics.

- Positive Z-N staining.

- Niacin production test - Positive.

- Nitrate reduction test - Positive.

Rapid growers are excluded in this study and no further steps are undertaken to identify them.

\section{Results}

Out of 102 clinically diagnosed pulmonary tuberculosis patients, 45, 73 and 72 cases were found to be positive for $\mathrm{AFB}$ by $\mathrm{ZN}$ staining, $\mathrm{AO}$ staining and culture techniques respectively. The $\mathrm{ZN}$ smear positivity rate and the $\mathrm{AO}$ smear positivity rate in this study was $44.1 \%(45 / 102)$ and $71.6 \%$ (73/102) respectively. The combined smear positivity using both the staining techniques was $72.5 \%(74 / 102)$.

Table 1 shows that scores are definitely higher by fluorescence microscopy: $73(20+28+16+9)$ positive as against $45(3+19+16+7)$ positive by the $\mathrm{ZN}$ method. The difference in the case-yields was found to be highly significant $\left(\chi^{2}=24.93, \mathrm{p}<0.001\right)$.

Disregarding the scores, $72(28+44)$ of 102 smears gave identical results. In other words, there was $70.6 \%$ agreement or $29.4 \%$ disagreement between $\mathrm{ZN}$ and AO.
Results that were identical are plotted on the diagonal. Scores higher by fluorescence microscopy are shown above the diagonal and those higher by ZN microscopy below it.

In $\mathrm{ZN}$ stained smears, $42(19+16+7)$ multibacillary and 3 paucibacillary cases were detected whereas in fluorochrome stained smears $53 \quad(28+16+9)$ multibacillary and 20 paucibacillary cases were detected.

43 out of 72 culture positive cases were diagnosed by $\mathrm{ZN}$ stained smear microscopy. 29 cases missed by $\mathrm{ZN}$ were detected by culture. There was agreement in 71/102 cases $(69.6 \%)$ and disagreement in 31/102 (30.4\%).

There was agreement in 97/102 cases (95.1\%) and disagreement in $5 / 102(4.9 \%)$ between fluorescence microscopy and culture.

On comparison against culture, the gold standard in the detection of the tubercle bacilli, the sensitivity and the predictive value of negative test of the $\mathrm{ZN}$ stain was much lower than those of AO stain (Table 2). The false positive results of the AO stain were slightly higher than that of $\mathrm{ZN}$. The false negative results of the $\mathrm{ZN}$ stain were much higher than that of AO. Combining the results of $\mathrm{ZN}$ and $\mathrm{AO}$, the efficiency was significantly increased than that of the individual stains.

Table 1: Correlation of conventional bright-field microscopy (Ziehl-Neelsen) and fluorescence microscopy (Auramine-O) gradewise.

\begin{tabular}{|c|c|c|c|c|c|c|c|c|}
\hline \multirow{8}{*}{ 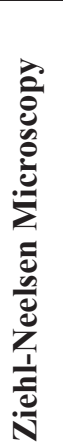 } & \multicolumn{7}{|c|}{ Fluorescence Microscopy } & \multirow{2}{*}{ Total } \\
\hline & Grading & NS & $\mathrm{D}$ & 1 & 2 & 3 & 4 & \\
\hline & NS & 25 & 3 & 13 & 5 & 0 & 0 & 46 \\
\hline & $\mathrm{D}$ & 0 & 0 & 5 & 4 & 1 & 1 & 11 \\
\hline & 1 & 0 & 1 & 2 & 0 & 0 & 0 & 3 \\
\hline & 2 & 0 & 0 & $\theta$ & 19 & 0 & 0 & 19 \\
\hline & 3 & 0 & 0 & 0 & $\cdots \cdots, 0$ & 15 & 1 & 16 \\
\hline & 4 & 0 & 0 & 0 & 0 & 0 & 7 & 7 \\
\hline \multicolumn{2}{|c|}{ Total } & 25 & 4 & 20 & 28 & 16 & 9 & 102 \\
\hline
\end{tabular}


Table 2: Evaluation of $\mathrm{ZN}, \mathrm{AO}$ and $\mathrm{ZN}+\mathrm{AO}$ microscopy against culture positive.

\begin{tabular}{|c|l|c|c|c|}
\hline S1. No. & \multicolumn{1}{|c|}{ Measures } & $\begin{array}{c}\text { ZN vs Culture } \\
(\mathbf{\%})\end{array}$ & $\begin{array}{c}\text { AO vs Culture } \\
(\mathbf{\%})\end{array}$ & $\begin{array}{c}\text { ZN+AO vs Culture } \\
(\%)\end{array}$ \\
\hline 1 & Sensitivity & 59.72 & 97.22 & 97.22 \\
\hline 2 & Specificity & 93.33 & 90 & 86.67 \\
\hline 3 & Predictive value of positive test & 95.55 & 95.89 & 94.59 \\
\hline 4 & Predictive value of negative test & 49.12 & 93.1 & 92.86 \\
\hline 5 & Percentage of false positive & 6.66 & 10 & 13.33 \\
\hline 6 & Percentage of false negative & 40.27 & 2.78 & 2.78 \\
\hline 7 & Efficiency & 69.6 & 85.29 & 94.11 \\
\hline
\end{tabular}

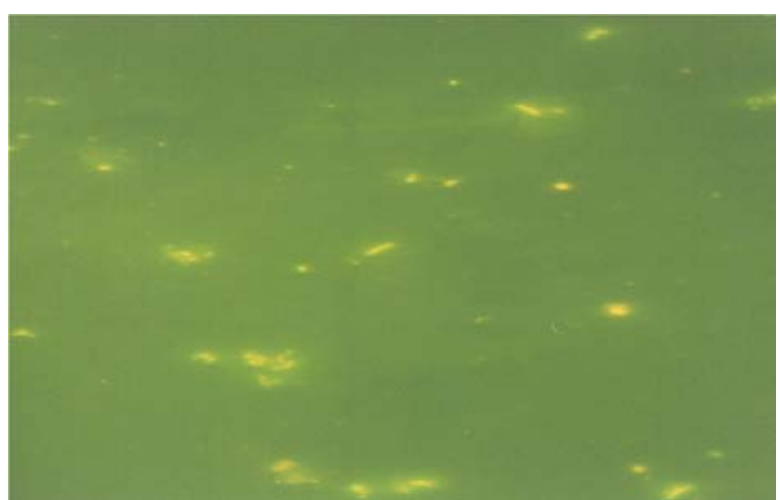

Fig 1: Fluorescent Auramine O stained sputum smear showing TB bacilli seen as yellow luminous organisms in a dark background (x400).

\section{Discussion}

Different smear microscopy results were achieved by Jain A et al ZN 32.7\%, AO 41.6\%, Githui et al ZN $65 \%$, AO 80\%, Ulukanligil et al ZN 67.6\%, AO 85.7\%, Prasanthi K and Kumari AR ZN 50\%, AO 69\%. 3,8,9,10 It was evident that $\mathrm{AO}$ method results scored higher than that of $\mathrm{ZN}$ method in all these studies as was the case in this study (ZN 44.1\%, AO 71.6\%). In this study, $\mathrm{AO}$ was found to be $27.46 \%$ more effective than $\mathrm{ZN}$ staining. This shows that fluorochrome staining of sputum smears in comparison to that of $\mathrm{ZN}$ staining is a better method of microscopy $\left(\chi^{2}=24.93, \mathrm{p}<0.001\right)$.

Detection of smear positive cases is the highest priority in any TB control programme, as these cases are infectious and contribute to transmission of disease. Though smear positivity correlates well with infectivity, much of the transmission occurs before the level of bacilli reach $10^{5} / \mathrm{ml}$ in the sputum ${ }^{10}$. ZN stain can detect bacilli when they are in the order of $10^{5} / \mathrm{ml}$ of the sputum whereas a more sensitive AO stain can detect in the order of $10^{4} /$ $\mathrm{ml}_{\text {of sputum }}{ }^{1}$. That fluorochrome stain is more efficient over ZN stain in detecting paucibacillary cases has been proved in this study. It could detect 20 paucibacillary cases whereas $\mathrm{ZN}$ detected only 3 of them. Failure to detect, and hence, to treat paucibacillary cases can

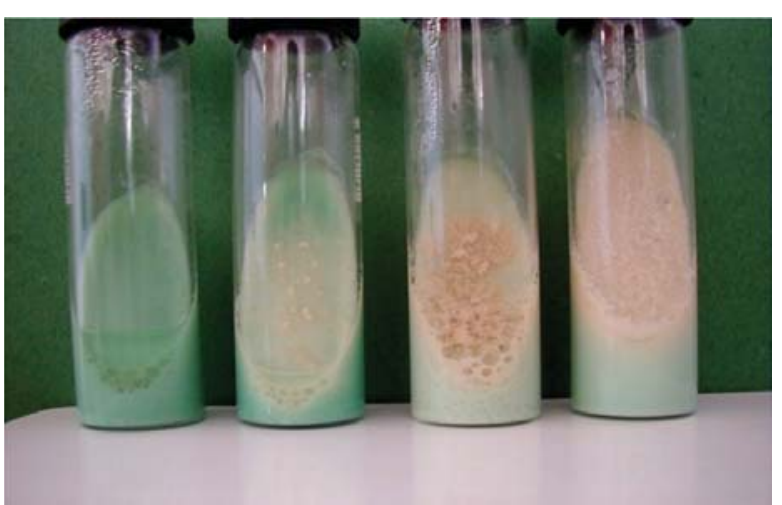

Fig 2: Growth of Mycobacterium tuberculosis on modified Lowenstein-Jensen media in McCartney bottles.

be effectively prevented by the use of fluorescence microscopy.

Kumar $\mathrm{N}$ et al had observed that $85 \%$ of the culture positive cases could be diagnosed by microscopy alone ${ }^{2}$. In this study, $59.7 \%(43 / 72)$ of the culture positive cases were diagnosed by ZN stained smear light microscopy. The figure increased significantly to $97.2 \%(70 / 72)$ of culture positive cases by AO stained smear fluorescent microscopy.

In this study, cultures were positive in $70.6 \%$ cases. On evaluation of the microscopic techniques by comparing them with the gold standard culture technique, it was found that in case of $\mathrm{ZN}$ stain there was agreement in $69.6 \%$ cases and disagreement in $30.4 \%$ whereas for AO stain there was agreement in $95.1 \%$ cases and disagreement in $4.9 \%$ cases. This proves that AO stain is a better method for its close comparability to the gold standard technique.

On evaluation of the microscopic techniques by comparing them with the gold standard culture technique, it was found that the sensitivity of $\mathrm{ZN}(59.72 \%)$ is much lower than that of AO (97.22\%). The result is similar to 
that of Jain A et al with sensitivity of $\mathrm{ZN}$ at $67.3 \%$ and AO $86.6 \%{ }^{3}$. The specificity of AO is quite high at $90 \%$ though it is lower than that of $\mathrm{ZN}$ at $93.3 \%$. The high specificity of fluorescent microscopy in the diagnosis of tuberculosis has been confirmed by Jain $\mathrm{A}$ et $\mathrm{al}^{3}$.

Jain A et al noted that around $40-50 \%$ of active pulmonary TB (culture positive) cannot be detected by $\mathrm{ZN}$ microscopy ${ }^{3}$. This was in concordance with the false negative results of $\mathrm{ZN}$ staining in this study $(40.27 \%)$. It is in sharp contrast to only $2.78 \%$ of false negative in case of AO stain. Hence, it is evident that a large number of cases missed out by $\mathrm{ZN}$ stain are easily detected by fluorochrome staining.

\section{Conclusion}

Sputum examination for the TB bacilli is usually conducted for patients clinically and/or radiologically suspected of pulmonary tuberculosis. However, the standard method of sputum examination, that is, $\mathrm{ZN}$ staining is not sensitive enough and a large number of these suspected cases miss diagnosis. Moreover, many cases remain unsuspected and do not seek treatment.

Fluorochrome stain is more efficient over $\mathrm{ZN}$ stain in detecting TB bacilli in sputum, especially the paucibacillary cases. Since screening is done under lower power of magnification (400x), fluorescence microscopy has been found to be less time consuming as compared to $\mathrm{ZN}$ method (1000x) in the diagnosis of tuberculosis. Hence, it has been advocated to be a method of choice where a large number of sputum smears are to be examined. The fluorescing bacilli are easily identifiable and cause less eye-strain.

Culture examination, no doubt, is more reliable but is time consuming, expensive and requires trained technical hands. The efficacy of fluorescence microscopy proved to be much higher than conventional light microscopy and comparable to that of culture. In this study, combining $\mathrm{ZN}$ and $\mathrm{AO}$ has been found to significantly improve sensitivity, predictive value of negative test, percentage of false negative and efficiency. Both staining methods are easy and cheap and may be used effectively instead of doing difficult, expensive and time consuming cultures.

\section{Acknowledgement}

We extend our thanks and gratitude to the Director, RIMS, Imphal, for allowing us to carry out this project in the Institution. We are also grateful to the Professor and Heads of Microbiology as well as Respiratory Diseases Departments of RIMS, Imphal, for providing us all the facilities available in the Departments while carrying out this work successfully.

\section{References}

1. Forbes BA, Sahm DF, Weissfeld AS. Role of Microscopy in the Diagnosis of Infectious Diseases. In: Bailey and Scotts' Diagnostic Microbiology. 10th edition. Missouri: Mosby, 1998: 134-49.

2. Kumar N, Tiwari NC, Verma K. AFB staining in cytodiagnosis of tuberculosis without classical features: A comparison of Ziehl-Neelsen and fluorescent methods. Cytopathology. 1998; 9(3): 208.

3. Jain A, Bhargava A, Agarwal SK. A comparative study of two commonly used staining techniques for acid fast bacilli in clinical specimens. Indian Journal of Tuberculosis. 2003; 49:161-2.

4. Ananthanarayanan R, Paniker CKJ. Mycobacterium-1 Tuberculosis. In: Textbook of Microbiology. 3rd edition. Madras: Orient Longman; 1988.p. 339-353.

5. Bose M, Varma M. Tuberculosis and MOTT in HIV/AIDS. In: Baveja UK, Sokhey J, editors. Manual on laboratory diagnosis of common opportunistic infections associated with HIV/ AIDS. New Delhi: Ministry of Health and Family Welfare, National AIDS Control Organisation; 1999.p. 88-99.

6. Xiaotian Z, Roberts GD. Diagnosis and Susceptibility Testing. In: David Schlossberg, editor. Tuberculosis and Non-tuberculous Mycobacterial Infections. Philadelphia: WB Saunders Company; 1999.p. 57-64.

7. Cruickshank R, Duguid JP, Swain RHA, Marmion BP. Mycobacterium. In: Medical Microbiology - The Practice of Medical Microbiology. Vol.2. 12th ed. Edinburgh: Churchill Livingstone; 1975.p. 387-91.

8. Githui W, Kitui F, Juma ES, Obwana DO, Mwai J, Kwamasga D. A comparative study on the reliability of the fluorescence microscopy and Ziehl-Neelsen method in the diagnosis of pulmonary tuberculosis. East Afr Med J. 1993; 70: 263-6.

9. Ulukanligil M, Aslan G, Tasci S. A comparative study on the different staining methods and number of specimens for the detection of acidfast bacilli. Mem Inst Oswaldo Cruz. 2000; 95: 855-8.

10. Prasanthi K, Kumari AR. Efficacy of fluorochrome stain in the diagnosis of pulmonary tuberculosis co-infected with HIV. Indian Journal of Medical Microbiology. 2005; 23(3):179-85. 